\title{
Islanding Protection and Islanding Detection in Low Voltage CIGRE Distribution Network with Distributed Generations*
}

\author{
Ghullam Mustafa Bhutto1,2\#, Ehsan Ali2,3, Jagdesh Kumar², Muhammad Akram Bhayo² \\ ${ }^{1}$ Department of Energy Technology, Aalborg University, Copenhagen, Denmark \\ ${ }^{2}$ Department of Electrical Engineering, Quaid-e-Awam University of Engineering, Science \& Technology, \\ Nawabshah, Pakistan \\ ${ }^{3}$ IM2NP, Aix-Marseille University, Marseille, France \\ Email:"gmu@et.aau.dk, "gmustafabhutto@yahoo.com, ehsanaliburiro@gmail.com, bhayoakram@yahoo.com \\ jagdesh.k24@gmail.com,
}

Received 5 April 2014; revised 11 May 2014; accepted 18 May 2014

Copyright (C) 2014 by authors and Scientific Research Publishing Inc.

This work is licensed under the Creative Commons Attribution International License (CC BY).

http://creativecommons.org/licenses/by/4.0/

(c) (i) Open Access

\section{Abstract}

The Power Quality (PQ), security, reliability etc., are the prime objectives of the power system. The protection is developed in such a way that it should be selective, fast, reliable and the cost effective. The study about the islanding protection in Low Voltage (LV) CIGRE distribution and networks like this has been proposed in this paper. This is achieved by developing the protection against the short circuit faults which might appear at the Medium Voltage (MV) bus. The protection of the network with significant penetration of the Distributed Generations (DGs) is a complicated process. The DG units which are directly connected to the grid such as synchronous or induction generators contribute large short power, whereas the DG units which are connected to the grid via inverters carry small amount of the short circuit power. This creates the problems in the protection of the network. If the proper protection coordination measures have not been taken, it might cause the mal-function of the protection devices which put the portion of the power network into the security threats. The selection of the islanding protection devices in this paper is made to protect the network against bi-directional currents at the time of short circuit fault. The LV CIGRE distribution network will enter into islanding if a fault is cleared at the MV bus by the proposed islanding protection devices. It is therefore, essential to detect the islanding in the CIGRE power network. The detection of the island in this network is another major objective of this paper. The detection of the island is proposed by using the technique which is based on the voltage phase angle difference. The simulations are carried out by using DIgSILENT power factory software version 15.0.

\footnotetext{
*1) Subject Classification: Smart Grids. 2) All authors are mutually agreed. 3) It’s the original work of all the authors. 4) We did not submit this manuscript before. 5) The research work carried out for this paper is related to the corresponding author's Ph.D. study, so some of the results or texts are directly or indirectly copied/used in this paper.

"Corresponding author.
}

How to cite this paper: Bhutto, G.M., Ali, E., Kumar, J. and Bhayo, M.A. (2014) Islanding Protection and Islanding Detection in Low Voltage CIGRE Distribution Network with Distributed Generations. Smart Grid and Renewable Energy, 5, $152-159$. 


\section{Keywords}

\section{Islanding Protection, Loss of Grid (LOG), Over Current Protection, Under Voltage Protection, Fuses, Relays, Island Detection (ID)}

\section{Introduction}

The large scale integration of DG units into the distribution grid presents several problems for the network. The islanding is one of the challenging problems in the electric network. Islanding, Loss of Grid (LOG) [1] is a situation where electrical system becomes electrically isolated from the rest of the power network and yet continues to be energized by the DG units connected to it. The main cause of the LOG is the occurrences of the faults in the network. If fault is not removed or a faulty portion is not quickly isolated from the healthy system, it will propagate into healthy parts of the network and will cause possible damages to the equipments available in the network. The urgent action is necessary to be taken in order to protect power network. The protection of the power network together with DG units and its coordination is complicated because of the bidirectional flow of the current and the difference in short circuit level of DG units and main power grid. This paper describes the new method of islanding protection.

It is also necessary to detect islanding in a power network as fast as possible as it might cause injuries to line worker and may create reclosing problems. Several island detection techniques have been proposed in the literature [2]-[6]. The island detection techniques can be generally divided into two categories: active methods and passive methods. The main passive techniques used for islanding detection include under/over frequency, vector shift, Rate Of Change Of Frequency (ROCOF) [7] [8], Under/Over Voltage, rate of change of voltage and power factor [9] etc. The main active techniques include reactive power Error Export Detection (REED) [10], fault level monitoring [11], and system impedance monitoring [5].

Passive methods are familiar for detecting LOG because of their simplicity and low cost. The ROCOF and vector shift methods are the most common methods used among the passive methods. These methods especially ROCOF method have some of the disadvantages that they do not properly differentiate between the island and transient conditions. Unnecessary tripping might occur in the case of sudden load changes, faults etc., if ROCOF method is used to detect islanding.

This paper describes the detection of LOG by using new technique which is based the voltage phase angle difference between the grid and the distribution system. This method is described in [7] but further details are added in this paper.

A test distribution network set up by CIGRE comprising Wind Turbine Generator (WTG), Photovoltaic (PV) solar generation units and sensitive power electronics based equipment such as Voltage Source Converters (VSCs) at different locations has been chosen for the study. The single line diagram of this distribution system is shown in Figure 1. Further details of CIGRE network can be found in [12].

The LV CIGRE distribution network modeled in DIgSILENT power factory software 15.0 is shown in Figure 2 together with appropriate protection devices.

Due to economic reasons, the protection of whole CIGRE network is mainly performed by the fuses. The detailed study about the protection of entire CIGRE network done by the author is presented in [13]. The LOG protection is performed by using fuse mainly in this study but the under voltage relay is also used to clear a fault if fuse is unable to detect faults in some conditions.

\section{Simulations Results}

The simulation results of the islanding protection and islanding detection are presented in the section given below.

\subsection{Islanding Protection}

A three phase fault with a fault impedance of zero ohm $\left(Z_{f}=0\right)$ is applied at time $\mathrm{t}=5 \mathrm{~s}$ on the MV bus (i.e., bus R0). There is a bi-directional flow of the current to the fault point in this condition. A fuse F0 as shown in 


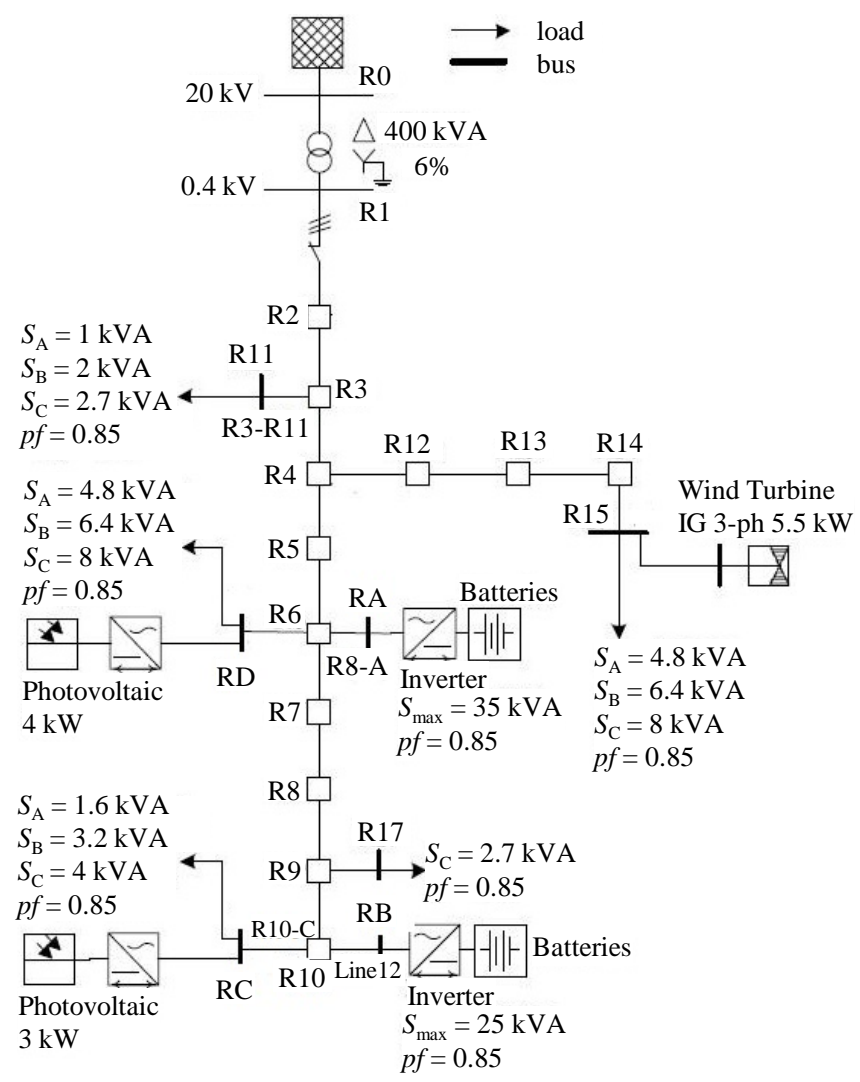

Figure 1. The single line diagram CIGRE distribution system test network [12].

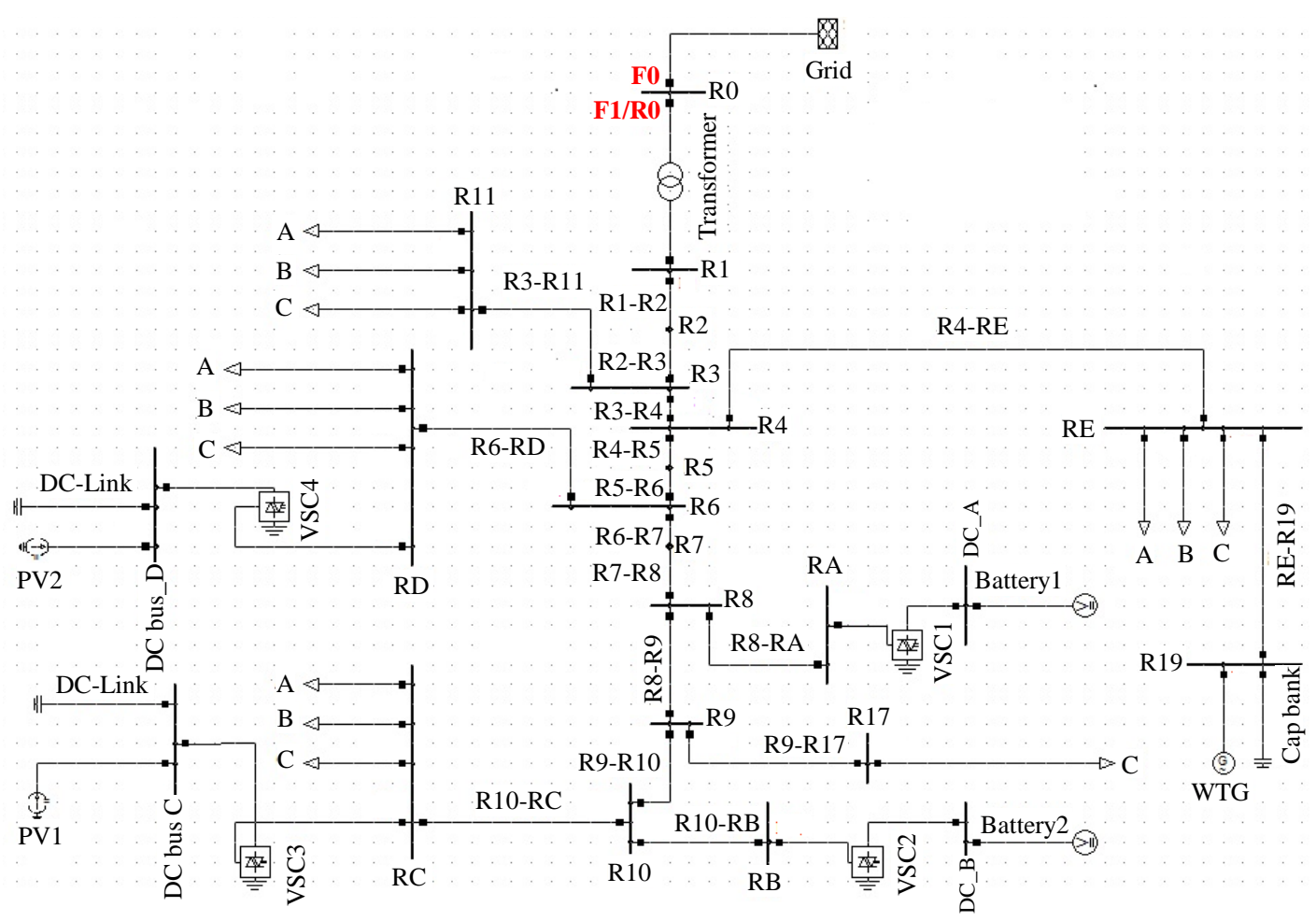

Figure 2. The CIGRE network modeled in DIgSILENT power factory together with protection devices. 
Figure 2 is used to disconnect the faulty section from the grid side. This enters the distribution network into island. Fuse F0 is selected to the next higher rated available than that of a fuse F1 to ensure the protection coordination. Fuse F1 does not clear a fault because it was selected for the protection in the forward direction (i.e., in the grid connected mode). The distribution network has a small amount of short circuit power when it is isolated from the grid, that's why fuse F1 does not clear a fault from the backward direction. The details about why fuse F1 do not clear a fault are described below with the help of simulation results.

A fault still exist in island portion of the network as it was only cleared from the grid side, therefore; to clear a fault in island portion of the network; a new approach of using the under voltage relay is introduced in this report. The under voltage relay R0 as shown in Figure 2 is used to isolate a fault from the DGs side of the network in this regard.

The current delivered by external gird which flows through a fuse F0 is shown in Figure 3. It can be seen in Figure 2 that grid delivers a huge amount of the current at the instant of a fault. When a fuse of $20 \mathrm{~A}$ (i.e., F0) sees this value of the current it blows within a very short duration as shown in Figure 3. Hence, a fault has been cleared from the grid side and should also be cleared from the DGs side very quickly.

The current seen by fuse F1 is shown in Figure 4. It can be seen in Figure 4 that the current which passes through the fusing element of fuse F1 increases at the instant of a fault and soon it decays. The peak in the current at the initiation of a fault is due to the current surge produced by DG units and it decays because of the current limiters used for these units. This current is coming from the DG side of the MG and passes through the 400 kVA transformer used between LV and MV buses. This current flowing through LV to the HV winding of the transformer (i.e., to the faulted point) passes through the transformer impedance. The current seen in Figure 4 is small because of the transformer impedance. This current in the steady state condition after the current peak does not reach to the value which melts fusing element.

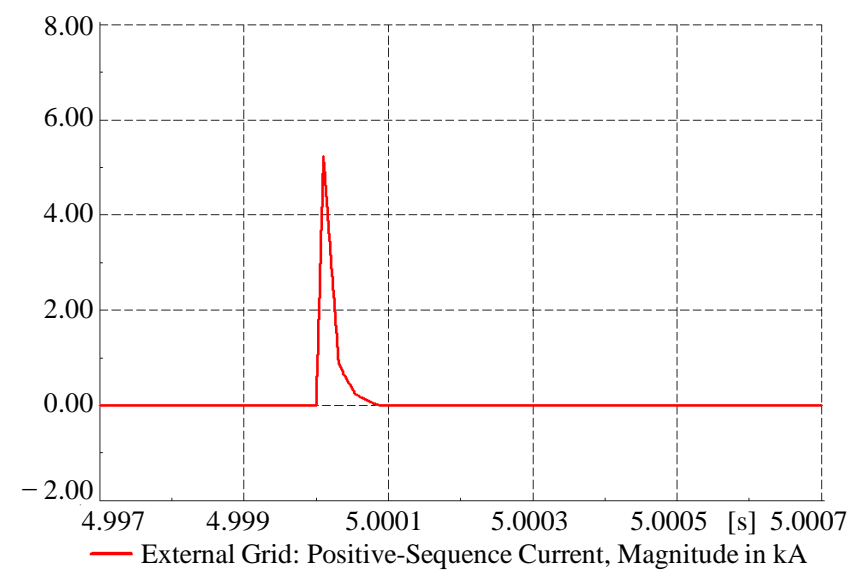

Figure 3. The external grid current.

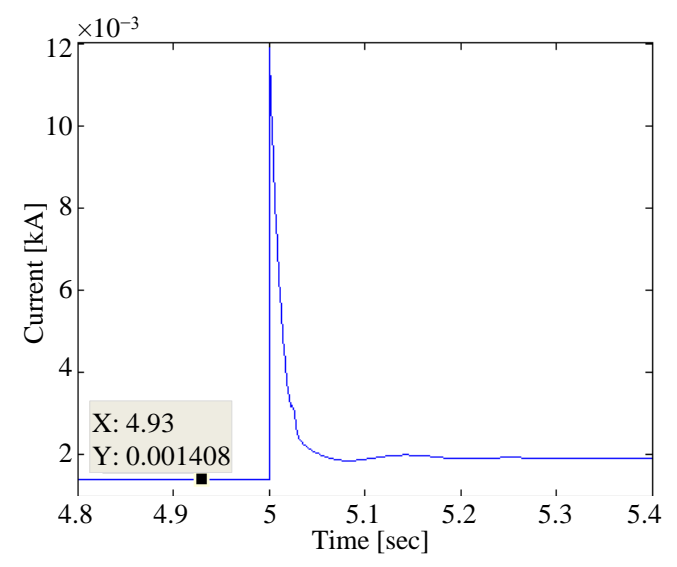

Figure 4. Current seen by fuse F1. 
The circuit breaker is used to clear this kind of fault in island portion of the network. The circuit breaker receives a trip signal from the under voltage relay R0. The circuit breaker named VD4 made by ABB is used in this regard. The minimum opening time of this circuit breaker lies between $33-60 \mathrm{~ms}$ [14]. The minimum opening time of circuit breaker used for this study is selected as $33 \mathrm{~ms}$. The time of the operation of a fuse F0 and circuit breaker receiving trip signal by under voltage relay R0 is shown in Figure 5.

The plot in Figure 5 demonstrates the clearance of the fault from both sides of the network and islanding protection has been achieved. The 1 on the plot in Figure 5 shows that a fuse operates in a normal operation and the contacts of the circuit breaker are in a close position whereas 0 displays that a fuse has been blown out and the breaker contacts are opened. It can be seen in Figure 5 that fuse F0 clears a fault from the grid side faster than the circuit breaker used on the DGs side of the network. A fuse blows faster because of severe current flowing across its melting element.

\subsection{Islanding Detection}

The detection of islanding in CIGRE network is performed by using a technique which is based on the phase angle difference between grid voltage and the DG side voltage. This islanding technique is described with the help of the block diagram shown in Figure 6.

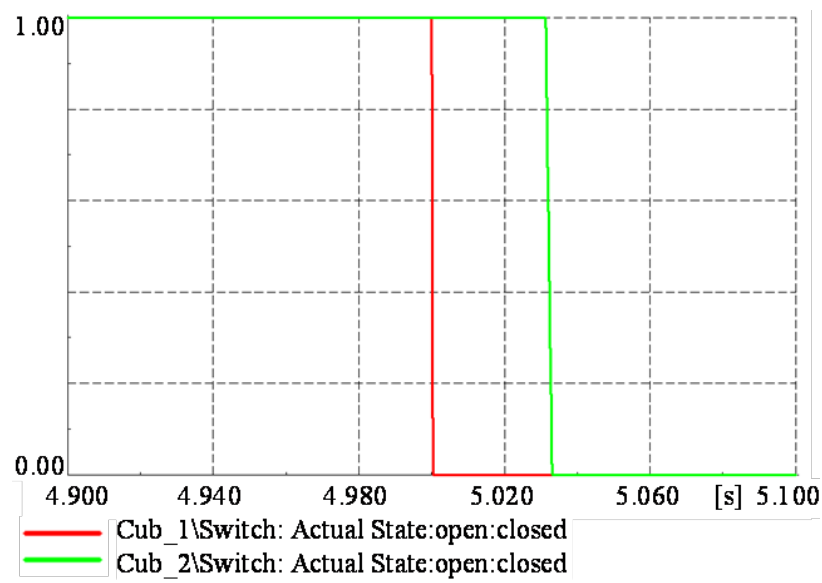

Figure 5. The time of operation of fuse and circuit breaker.

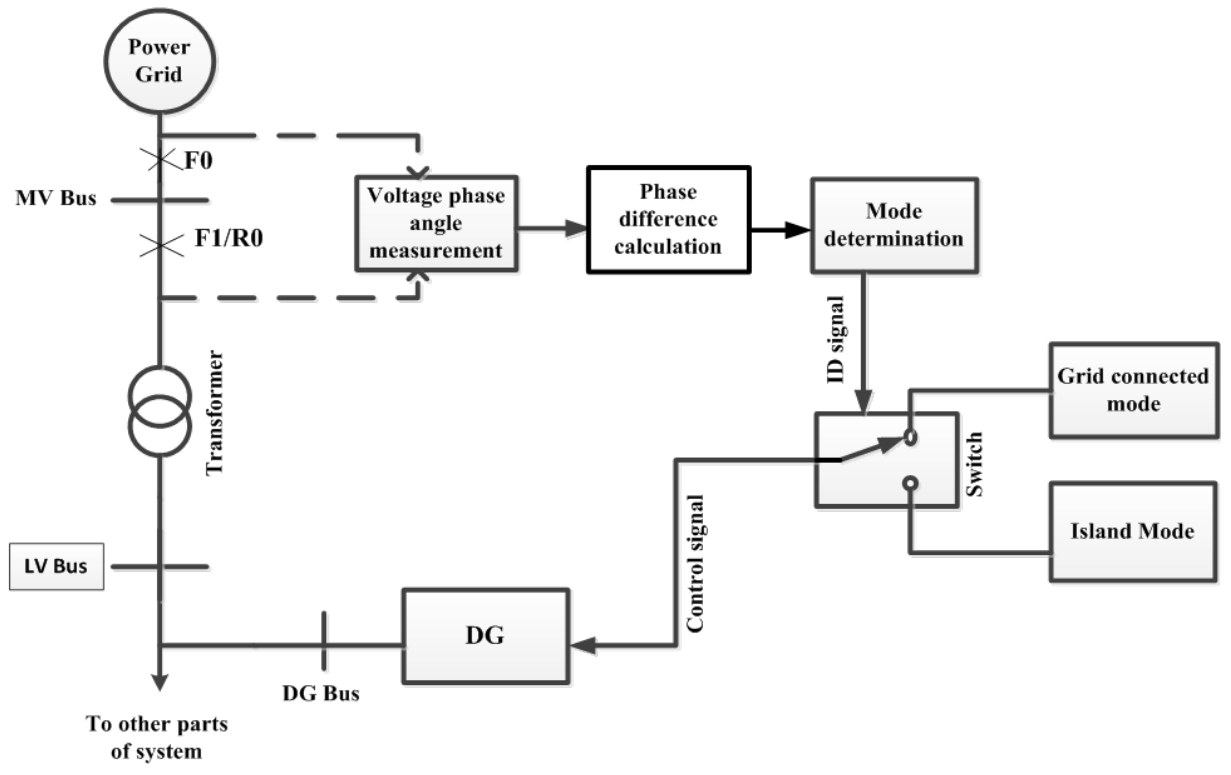

Figure 6. The block diagram of the island detection technique and control mode determination. 
The simple block of power network is shown in Figure 6. It can be seen in Figure 6 that the phase angles of the voltages at the grid side and DG side are measured and difference of the phases is calculated. The network changes its mode of operation from the grid connected to islanding according to the difference of the voltage phase angles. The island is detected in the network if there is a phase angle difference between the two voltages as shown in Figure 7.

It can be seen in Figure 7 that the phase angle difference of the voltages at the mentioned points in Figure 6 is zero in the normal operating conditions. This difference of the voltage phase angles changes when fuse F0 is blown out.

The Island Detection (ID) signal is shown in Figure 8. This figure describes that the system is connected to the grid when there is no difference between voltage phase angles. The network switches to island mode when the difference in the voltage phase angles is appeared. Hence, LOG is detected at $5.0001 \mathrm{~s}$ in the CIGRE low voltage network.

This method of LOG detection performs well during sudden switching/variation of the loads and in the transient conditions somewhere in other parts of the CIGRE network. To see the effects on the transients and check whether this method may not cause false detection in case of the fault, the study is performed by applying a 3phase fault on bus R11 at the time equal to $\mathrm{t}=5 \mathrm{~s}$. The voltage phase angles in this case can be read in Figure 9 and the ID signal is shown in Figure 10.

It can be seen in Figure 9 that voltage phase angles at the time of short circuit fault i.e., $\mathrm{t}=5 \mathrm{~s}$ changes but their difference is zero. Hence, system remains connected to the grid and there is no false detection by using this method as shown in Figure 10. This is the main advantage of this method over other methods of LOG detection.

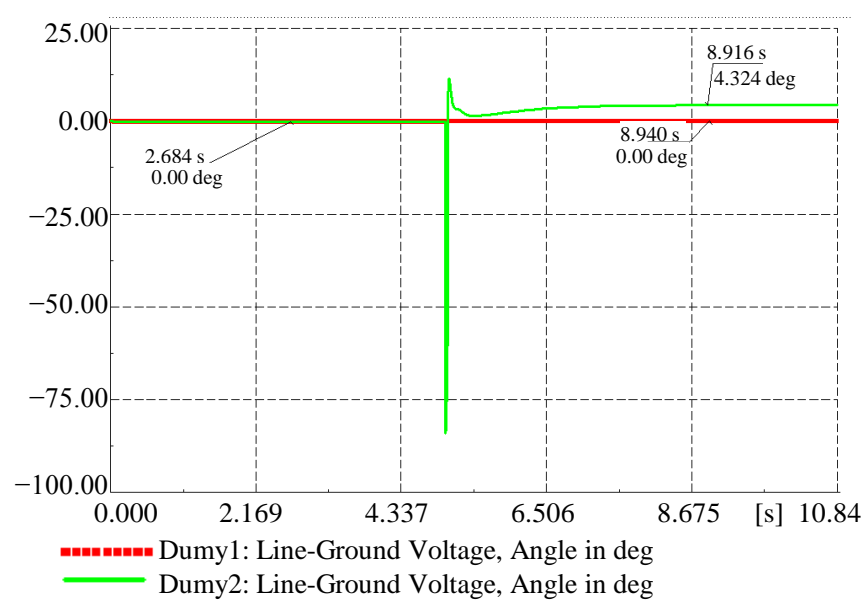

Figure 7. Phase angle difference between the voltages.

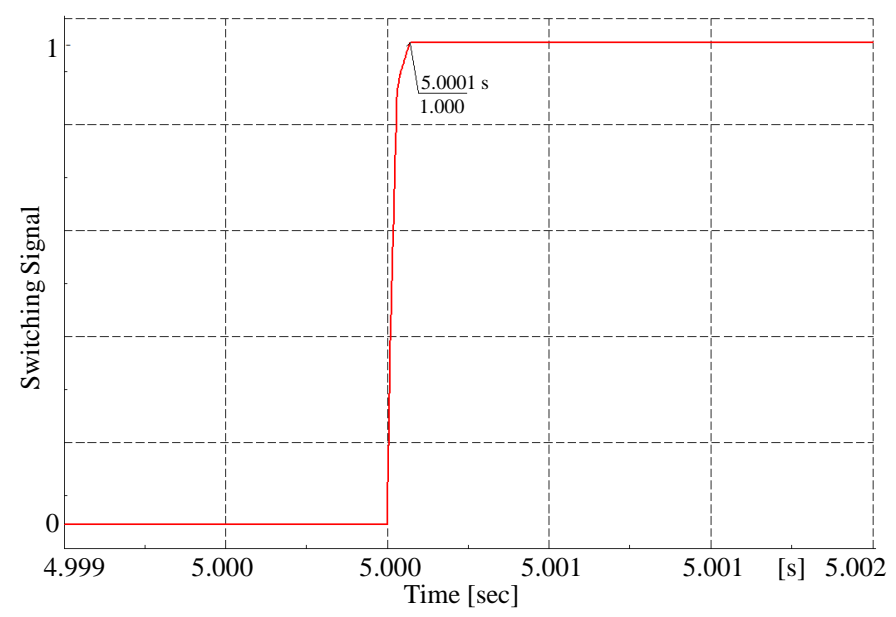

Figure 8. Island Detection (ID) signal. 


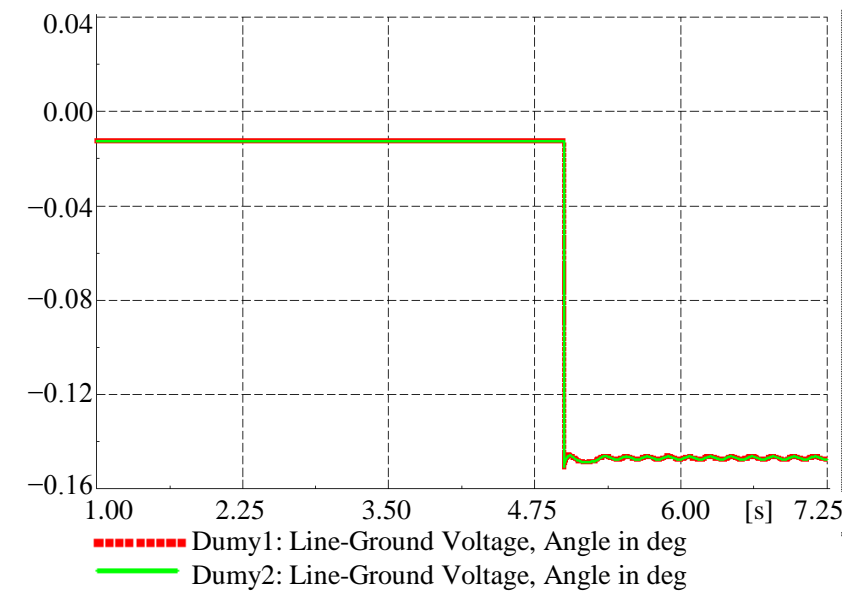

Figure 9. Phase angle difference between the voltages.

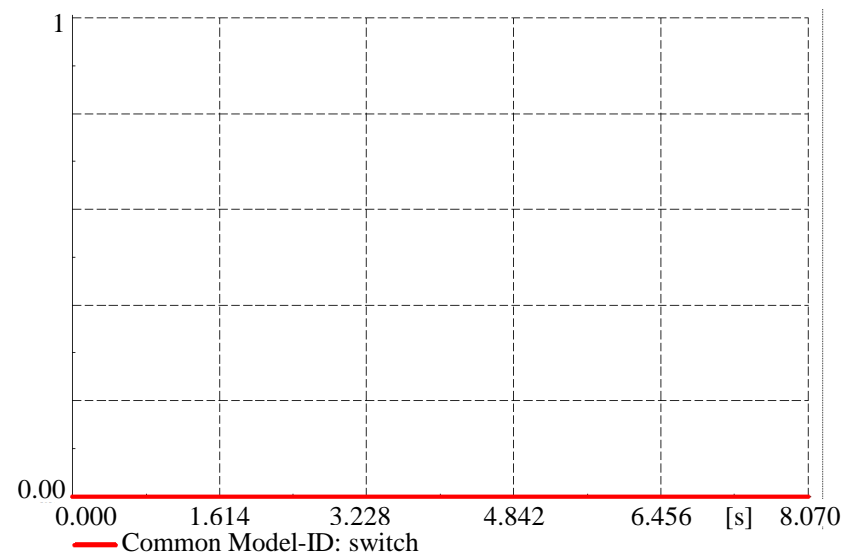

Figure 10. ID signal.

\section{Conclusion}

The protection against the bi-directional fault current in low voltage CIGRE network has been described in this paper by using current based and voltage based protection devices. The overview of the available island detection techniques is presented and the detection of LOG is proposed by the technique which is based on voltage phase angle differences. The working of this method has been validated by various simulations plots and its main advantages over the other methods are also described with the help of the plots. The problems of voltage and frequency in island Micro Grid (MG) will be tackled in future.

\section{Acknowledgements}

We are thankful to Kai Strunz for providing the CIGRE test network for LV distribution network. I, Ghullam Mustafa am thankful to Aalborg University and Quaid-E-Awam University of Engineering Sciences and Technology, Nawabshah, Sindh, Pakistan for the support and funding.

\section{References}

[1] Jenkings, N., Allan, R., Crossley, P., Kirschen, D. and Strbac, G. (2000) Embedded Generation (IEE Power and Energy Series 31), The Institution of Engineering and Technology, London.

[2] Ropp, M., Aaker, K., Haigh, J. and Sabhah, N. (2000) Using Power Line Carrier Communications to Prevent Islanding. Proceedings of 28th IEEE Photovoltaic Specialists Conference, Anchorage, 15-22 September 2000, 1675-1678. http://dx.doi.org/10.1109/PVSC.2000.916224

[3] Xu, W., Zhang, G., Li, C., Wang, W., Wang, G. and Kliber, J. (2007) A Power Line Signaling Based Technique for 
Anti-Islanding Protection of Distributed Generators_-Part I: Scheme and Analysis. IEEE Transactions on Power Delivery, 22, 1758-1766. http://dx.doi.org/10.1109/TPWRD.2007.899618

[4] Wang, G., Kliber, J., Zhang, G., Xu, W., Howell, B. and Palladino, T. (2007) A Power Line Signaling Based Technique for Anti-Islanding Protection of Distributed Generators-Part II: Field Test Results. IEEE Transactions on Power Delivery, 22, 1767-1772. http://dx.doi.org/10.1109/TPWRD.2007.899620

[5] O’Kane, P. and Fox, B. (1997) Loss of Mains Detection for Embedded Generation by System Impedance Monitoring. 6th International Conference on Developments in Power System Protection, Nottingham, 25-27 March 1997, 95-98. http://dx.doi.org/10.1049/cp:19970037

[6] Usta, O., Redfern, M.A. and Barrett, J.I. (1994) Protection of Dispersed Storage and Generation Units against Islanding. Proceedings of 7th Electrotechnical Conference on Mediterranean, Antalya, 12-14 April 1994, 976-979. http://dx.doi.org/10.1109/MELCON.1994.380938

[7] Affonso, C.M., Freitas, W., Xu, W. and da Silva, L.C.P. (2005) Performance of ROCOF Relays for Embedded Generation Applications. IEE Proceedings of Generation, Transmission and Distribution, 10 January 2005, 109-114.

[8] Ding, X., Crossley, P.A. and Morrow, D.J. (2007) Islanding Detection for Distributed Generation. Journal of Electrical Engineering \& Technology, 2, 19-28. http://dx.doi.org/10.5370/JEET.2007.2.1.019

[9] Salman, S.K., King, D.J. and Weller, G. (2001) New Loss of Mains Detection Algorithm for Embedded Generation Using Rate of Change of Voltage and Changes in Power Factors. Proceedings of 7th International Conference on Developments in Power System Protection, Amsterdam, 9-12 April 2001, 82-85.

[10] Ding, X., Crossley, P.A. and Morrow, D.J. (2007) Islanding Detection for Distributed Generation. Journal of Electrical Engineering \& Technology, 2, 19-28. http://dx.doi.org/10.5370/JEET.2007.2.1.019

[11] Cooper, C.B. (1989) Standby Generation Problems and Prospective Gains from Parallel Running. Proceedings of International Power System Protection Conference, Singapore, 1-6.

[12] CIGRE Task Force C6.04.02 (2011) Benchmark Systems for Network Integration of Renewable Energy Resources, Version 7.

[13] Mustafa, G., Bak-Jensen, B., Bak, C.L. and Pillai, J.R. (2013) Protection of Low Voltage CIGRE Distribution Network. Smart Grids and Renewable Energy, 4, 489-500.

[14] Medium Voltage Products, VD4 Medium Voltage Vacuum Circuit Breakers (12 - 36 kV). www.abb.com 
Scientific Research Publishing (SCIRP) is one of the largest Open Access journal publishers. It is currently publishing more than 200 open access, online, peer-reviewed journals covering a wide range of academic disciplines. SCIRP serves the worldwide academic communities and contributes to the progress and application of science with its publication.

Other selected journals from SCIRP are listed as below. Submit your manuscript to us via either submit@scirp.org or Online Submission Portal.
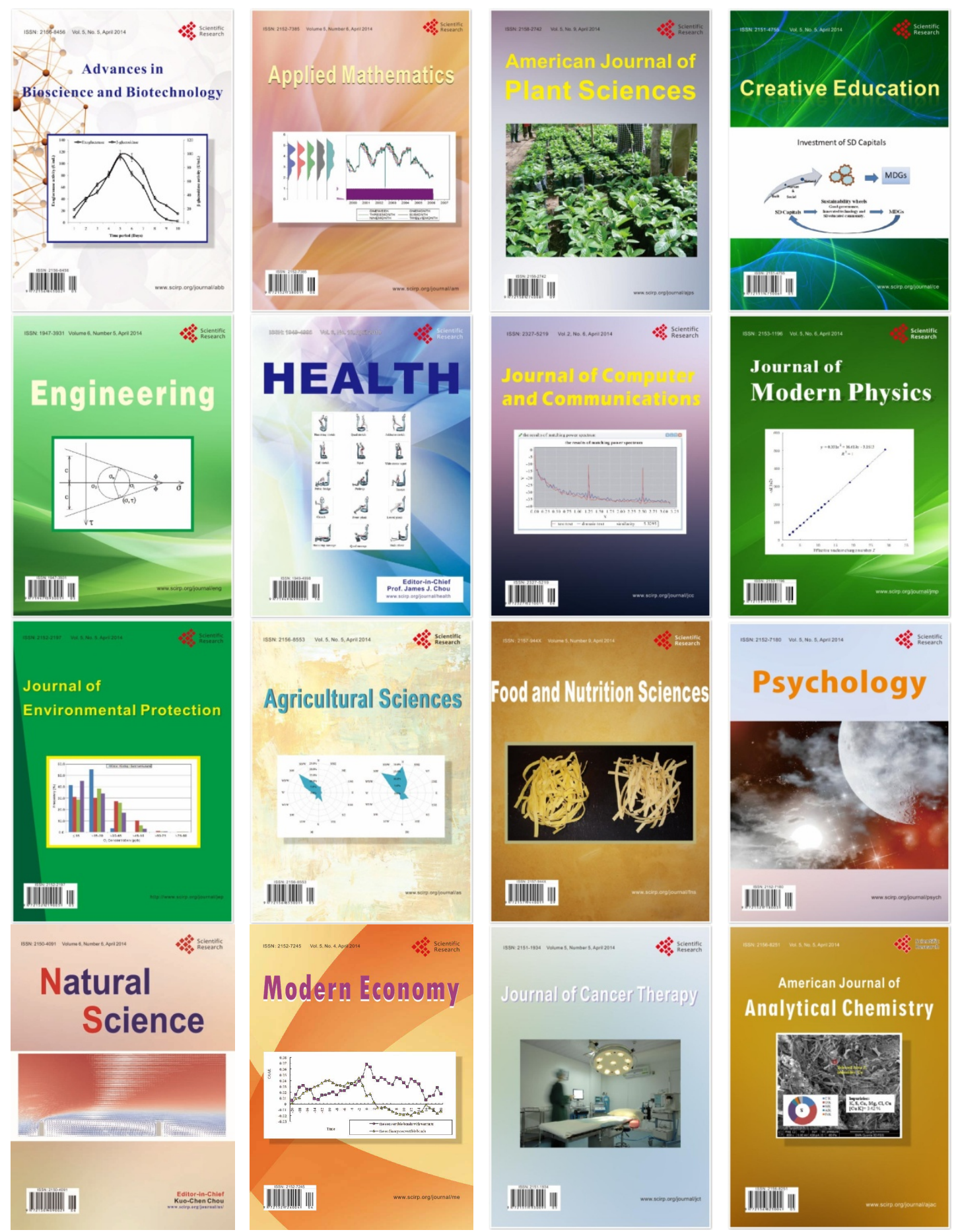